Pacific Journal of Mathematics

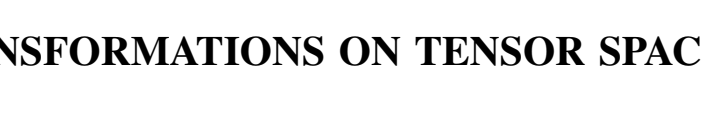




\section{TRANSFORMATIONS ON TENSOR SPACES}

\section{ROY WESTWICK}

In this paper we consider those linear transformations from one tensor product of vector spaces to another which carry nonzero decomposable tensors into nonzero decomposable tensors. We obtain a general decomposition theorem for such transformations. If we suppose further that the transformation maps the space into itself then we have a complete structure theorem in the following two cases: (1) the transformation is onto, and (2) the field is algebraically closed and the tensor space is a product of finite dimensional vector spaces. The main results are contained in Theorems 3.5 and 3.8 which state that the transformation $T: U_{1} \otimes \cdots \otimes U_{n} \rightarrow U_{1} \otimes \cdots \otimes U_{n}$ has the form $T\left(x_{1} \otimes \cdots \otimes x_{n}\right)=T_{1}\left(x_{\pi(1)}\right) \otimes \cdots \otimes T_{n}\left(x_{\pi(n)}\right)$ where $T_{i}: U_{\pi(i)} \rightarrow U_{i}$ are nonsingular and $\pi$ is a permutation. Case (2) generalizes a theorem of Marcus and Moyls.

Let $F$ be a field and $\left\{U_{a}: a \in A\right\}$ be a finite set of vector spaces over $F$. Let $(U, t)=\left(\otimes\left(U_{a}: a \in A\right), t\right)$ be a tensor product. Then $U$ is a vector space over $F, t: \Pi\left(U_{a}: a \in A\right) \rightarrow U$ is multilinear and, for any vector space $V$ over $F$ and multilinear map $f: \Pi\left(U_{a}: a \in A\right) \rightarrow V$, there is a unique linear transformation $g: U \rightarrow V$ such that $g \cdot t=f$. The decomposable tensors of $U$ are defined to be the vectors $t\left(\Pi\left(u_{a}: a \in A\right)\right)$, denoted by $\otimes\left(u_{a}: a \in A\right)$, where $u_{a} \in U_{a}$ for $a \in A$.

The proofs of the main theorems are based on the purely combinatorial results of the following section.

2. Adjacency preserving functions. In this section we define the adjacency preserving functions and find a decomposition theorem for them.

Let $A$ be a nonempty finite set and for each $a \in A$ let $S_{a}$ be a nonempty set. If $J$ is a nonempty subset of $A$ we let $p_{J}$ denote the projection of $\Pi\left(S_{a}: a \in A\right)$ onto $\Pi\left(S_{a}: a \in J\right)$. If $J=\{a\}$ we write $p_{a}$ for $p_{J}$.

For each $J \subseteq A$ we define an equivalence relation, denoted by $\equiv(\bmod J)$, on $\Pi\left(S_{a}: a \in A\right)$ by setting $x \equiv y(\bmod J)$ if and only if $p_{a}(x)=p_{a}(y)$ for all $a \in A \backslash J$. If $X \subseteq \Pi\left(S_{a}: a \in A\right)$ is a nonempty subset of equivalent elements relative to $\equiv(\bmod J)$ then we call $X$ a $J$-subset. If $J=\{a\}$ is a singleton we use $a$-subset for $J$-subset. We note the following

2.1. Lemma. A subset $X \subseteq \Pi\left(S_{a}: a \in A\right)$ is an equivalence class relative to $\equiv(\bmod J)$ if and only if $p_{J}(X)=\Pi\left(S_{a}: a \in J\right)$ and $p_{a}(X)$ 
is a singleton for each $a \in A \backslash J$.

The equivalence classes will be called maximal $J$-subsets.

On the set of subsets of $\Pi\left(S_{a}: a \in A\right)$ we define, for each $J \subseteq A$, the relation $\operatorname{adj}(\bmod J)$ by setting $X \operatorname{adj} Y(\bmod J)$ if and only if $X$ and $Y$ are $J$-subsets and for precisely one $a \in A \backslash J$ we have $p_{a}(X) \neq$ $p_{a}(Y)$. If $x, y \in \Pi\left(S_{a}: a \in A\right)$ and $\{x\} \operatorname{adj}\{y\}(\bmod \phi)$ then we say that $x$ and $y$ are adjacent. The relation $\operatorname{adj}(\bmod J)$ is symmetric but neither reflexive non transitive.

2.2. Lemma. Let $J \subseteq A$ and let $X$ and $Y$ be distinct maximal $J$-subsets of $\Pi\left(S_{a}: a \in A\right)$. Then there is a finite sequence $X_{1}, \cdots, X_{n}$ of maximal $J$-subsets such that $X=X_{1}, Y=X_{n}$, and $X_{i}$ adj $X_{i+1}(\bmod J)$ for $i=1, \cdots, n-1$.

Proof. Let $a_{1}, \cdots, a_{n}$ be the distinct elements of $A \backslash J$ for which $p_{a_{i}}(X) \neq p_{a_{i}}(Y)$. Then the maximal $J$-subsets $X_{i}$ for which $P_{a_{j}}\left(X_{i}\right)=$ $p_{a_{j}}(Y)$ for $j \leqq i$ and $p_{a_{j}}\left(X_{i}\right)=p_{a_{j}}(X)$ for $j>i$ will suffice.

2.3. Definition. A function from one cartesian product of sets into another is an adjacency preserving function if and only if the images of adjacent elements are adjacent.

2.4. Lemma. Let $f: \Pi\left(S_{a}: a \in A\right) \rightarrow \Pi\left(R_{b}: b \in B\right)$ be an adjacency preserving function. For each $a \in A$ let $S_{a}$ contain at least three elements. Then there is a function $\sigma: A \rightarrow B$ such that for any $c \in A$ and any maximal c-subset $X$ of $\Pi\left(S_{a}: a \in A\right), f(X)$ is a $\sigma(c)$-subset of $\Pi\left(R_{b}: b \in B\right)$.

Proof. Let $c \in A$ and let $X$ be a maximal $c$-subset of $\Pi\left(S_{a}: a \in A\right)$. Then $f(X)$ is a $d$-subset of $\Pi\left(R_{b}: b \in B\right)$ for some $d \in B$, where $d$ depends on $c$ and $X$. For, let $x_{1}$ and $x_{2}$ be distinct elements of $X$ and let $d \in B$ be that element of $B$ for which $p_{d}\left(f\left(x_{1}\right)\right) \neq p_{d}\left(f\left(x_{2}\right)\right)$. Then, for any $x \in X, p_{d}(f(x))$ differs from at least one of the $p_{d}\left(f\left(x_{i}\right)\right)$ and so $p_{b}(f(x))$ is independent of $x \in X$ for $b \neq d$. Therefore $f(X)$ is a $d$-subset. We let $\sigma(c, X)=d$. We show that $\sigma(c, X)$ is independent of the maximal $c$-subset $X$. Suppose the contrary. Then, from Lemma 2.2 it follows easily that there is a pair of maximal $c$-subsets $X$ and $Y$ for which $X$ adj $Y(\bmod \{c\})$ and $\sigma(c, X)=d_{1} \neq d_{2}=\sigma(c, Y)$. Let $c^{\prime}$ be the unique element of $A$ for which $p_{c^{\prime}}(X) \neq p_{c^{\prime}}(Y)$. Let $q: X \rightarrow Y$ be defined on each $x \in X$ by $p_{a}(q(x))=p_{a}(x)$ if $a \neq c^{\prime}$ and $p_{c^{\prime}}(q(x))=p_{c^{\prime}}(Y)$. Then $q$ is one-to-one, onto, and for each $x \in X$ the pair $x$ and $q(x)$ are adjacent. Since $S_{c}$ has at least three elements there are at least two elements $x \in X$ such that $p_{d_{1}}(f(x)) \neq p_{d_{1}}(f(Y))$, 
and of these, at least one for which $p_{d_{2}}(f(q(x))) \neq p_{d_{2}}(f(X))$. Now, for any $x \in X$ satisfying both of these inequalities, $f(x)$ and $f(q(x))$ are not adjacent, contrary to the hypothesis on $f$. Therefore $\sigma(c, X)$ is independent of the $c$-subset $X$ and so $\sigma(c)=\sigma(c, X)$ is a well defined function satisfying the conclusion of the lemma.

2.5. Theorem. Let $f: \Pi\left(S_{a}: a \in A\right) \rightarrow \prod\left(R_{b}: b \in B\right)$ be an adjacency preserving function and suppose that each $S_{a}$ contains at least three elements. Then there is a partition of $A$ into subsets $A_{1}, \cdots, A_{k}$ and distinct elements $b_{1}, \cdots, b_{k}$ of $B$ such that for each $i=1, \cdots, k$ there is a function $f_{i}: \Pi\left(S_{a}: a \in A_{i}\right) \rightarrow R_{b_{i}}$ satisfying $p_{b_{i}} \cdot f=f_{i} \cdot p_{A_{i}}$. Furthermore, the image of $\Pi\left(S_{a}: a \in A\right)$ under $f$ is the set $\Pi\left(Q_{b}: b \in B\right)$ where $Q_{b}$ is the image of $\Pi\left(S_{a}: a \in A\right)$ under $\left(p_{b} \cdot f\right)$.

Proof. Let $\sigma$ be given as in Lemma 2.4. Let $\left\{b_{1}, \cdots, b_{k}\right\}=\sigma(A)$ and let $A_{i}=\sigma^{-1}\left(b_{\imath}\right)$. Then $A_{1}, \cdots, A_{k}$ is a partition of $A$. Let $J$ be one of the $A_{i}$ and $b$ the corresponding $b_{i}$. Let $X$ be a maximal $J$-subset of $\Pi\left(S_{a}: a \in A\right)$. We define $f_{X}: \Pi\left(S_{a}: a \in J\right) \rightarrow R_{b}$ by

$$
f_{X}=p_{b} \cdot f \cdot\left(p_{J} \mid X\right)^{-1} .
$$

Then $f_{X}$ is well defined since $\left(p_{J} \mid X\right)$ is a one-to-one function from $X$ onto II $\left(S_{a}: a \in J\right)$. We prove that $f_{X}=f_{Y}$ for any two maximal $J$-subsets $X$ and $Y$. Suppose the contrary. Then, from Lemma 2.2, it follows that we can choose maximal $J$-subsets $X$ and $Y$ such that $X \operatorname{adj} Y(\bmod J)$ and $f_{X} \neq f_{Y}$. Let $a^{\prime} \in A \backslash J$ be that element for which $p_{a^{\prime}}(X) \neq p_{a^{\prime}}(Y)$. Choose $s \in \Pi\left(S_{a}: \alpha \in J\right)$ such that $f_{X}(s) \neq f_{Y}(s)$. Let $x=\left(p_{J} \mid X\right)^{-1}(s)$ and $y=\left(p_{J} \mid Y\right)^{-1}(s)$. Then $x \in X$ and $y \in Y$ are a pair of adjacent elements of $\Pi\left(S_{a}: a \in A\right)$. If we let $b^{\prime}=\sigma\left(a^{\prime}\right)$ then $b^{\prime} \neq b$ since $a^{\prime} \notin J=\sigma^{-1}(b)$. Therefore, $f(x)$ and $f(y)$ are adjacent and $b^{\prime}$ is the only element of $B$ for which $p_{b^{\prime}}(f(x)) \neq p_{b^{\prime}}(f(y))$. But $p_{b}(f(x))=$ $f_{X}(s) \neq f_{Y}(s)=p_{b}(f(y))$, a contradiction.

For each $i$ we set $f_{i}=f_{X}$ where $X$ is any maximal $A_{i}$-subset of $\Pi\left(S_{a}: a \in A\right)$. Then, if $x \in \Pi\left(S_{a}: a \in A\right)$, we choose a maximal $A_{i^{-}}$ subset $X$ containing $x$ and note that

$$
\left(f_{i} \cdot p_{A_{i}}\right)(x)=\left(p_{b_{i}} \cdot f \cdot\left(p_{A_{i}} \mid X\right)^{-1}\right)\left(p_{A_{i}}(x)\right)=\left(p_{b i} \cdot f\right)(x) .
$$

If $b \in \sigma(A)$ then the image of $\Pi\left(S_{a}: a \in A\right)$ under $\left(p_{b} \cdot f\right)$ consists of one element of $R_{b}$. In fact, suppose $x$ and $y$ are adjacent elements of II $\left(S_{a}: a \in A\right)$. Then $f(x)$ and $f(y)$ are adjacent and the element $b^{\prime} \in B$ for which $p_{b^{\prime}}(f(x)) \neq p_{b^{\prime}}(f(y))$ is in $\sigma(A)$. Then $\left(p_{b} \cdot f\right)(x)=$ $\left(p_{b} \cdot f\right)(y)$.

It is clear that $f\left(\Pi\left(S_{a}: a \in A\right) \subseteq \Pi\left(Q_{b}: b \in B\right)\right.$. To show that we 
have equality, suppose that $x \in \Pi\left(Q_{b}: b \in B\right)$. Choose $y_{b} \in \Pi\left(S_{a}: a \in A\right)$ such that $\left(p_{b} \cdot f\right)\left(y_{b}\right)=p_{b}(x)$. Choose $y \in \Pi\left(S_{a}: a \in A\right)$ such that $p_{A_{i}}(y)=$ $p_{A_{i}}\left(y_{b_{i}}\right)$ for $i=1, \cdots, k$. This can be done since the sets $A_{1}, \cdots, A_{k}$ are pairwise disjoint. If $b \notin \sigma(A)$ then $\left(p_{b} \cdot f\right)(y)=p_{b}(x)$ since the image is independent of $y$. For $b_{i}$ we have $\left(p_{b_{i}} \cdot f\right)(y)=f_{A_{i}}\left(p_{A_{i}}(y)\right)=$ $f_{A_{i}}\left(p_{A_{i}}\left(y_{b_{i}}\right)\right)=p_{b_{i}}\left(f\left(y_{b_{i}}\right)\right)=p_{b_{i}}(x)$. Therefore $f(y)=x$, and this completes the proof.

3. The preservers of decomposable tensors. In this section we require the

3.1. Lemma. Let $U=\otimes\left(U_{a}: a \in A\right)$ be a tensor product where the $U_{a}$ are vector spaces over a field $F$. Let $x_{a}, x_{a}^{\prime} \in U_{a}$ for $a \in A$. Then

(1) $\otimes\left(x_{a}: a \in A\right)=0$ if and only if $x_{a}=0$ for some $a \in A$.

(2) If $x=\bigotimes\left(x_{a}: a \in A\right)$ and $x^{\prime}=\bigotimes\left(x_{a}^{\prime}: a \in A\right)$ are nonzero decomposable tensors then,

(a) $\langle x\rangle=\left\langle x^{\prime}\right\rangle$ if and only if $\left\langle x_{a}\right\rangle=\left\langle x_{a}^{\prime}\right\rangle$ for $a \in A$.

(b) $x+x^{\prime}$ is a decomposable tensor if and only if $\left\langle x_{a}\right\rangle=$ $\left\langle x_{a}^{\prime}\right\rangle$ for all except possibly one $a \in A$.

Proof. The statements (1) and (2)(a) are elementary properties of $U$. The sufficiency of the condition in (2)(b) is clear. We prove the necessity of this condition by the following indirect argument. Suppose $x+x^{\prime}=\otimes\left(y_{a}: a \in A\right)$ where $\left\langle x_{b}\right\rangle \neq\left\langle x_{b}^{\prime}\right\rangle$ and $\left\langle x_{c}\right\rangle \neq\left\langle x_{c}^{\prime}\right\rangle$ for a pair of indicies $b$ and $c$. We may suppose that $\left\langle y_{b}\right\rangle \neq\left\langle x_{b}\right\rangle$. We define a function $f: \Pi\left(U_{a}: a \in A\right) \rightarrow F$ as follows. For each $a \in A$ we choose a linear functional $f_{a} \in \mathscr{L}\left(U_{a}, F\right)$ such that

(1) $f_{a}\left(x_{a}\right) \neq 0$ for all $a \in A$,

(2) $f_{b}\left(y_{b}\right)=f_{c}\left(x_{c}^{\prime}\right)=0$,

and set $f(u)=\Pi\left(f_{a}\left(p_{a}(u)\right): a \in A\right)$. Then $f$ is multilinear and it induces a linear transformation $f^{\prime}: \otimes\left(U_{a}: a \in A\right) \rightarrow F$. But $0=f^{\prime}(y)=$ $f^{\prime}\left(x+x^{\prime}\right)=f^{\prime}(x)+f^{\prime}\left(x^{\prime}\right)=f^{\prime}(x) \neq 0$, which is impossible.

Throughout the rest of this section we let $U=\otimes\left(U_{a}: a \in A\right)$ and $W=\otimes\left(W_{b}: b \in B\right)$ where the $U_{a}$ and $W_{b}$ are vector spaces over a field $F$. We also assume that $\operatorname{dim}\left(U_{a}\right) \geqq 2$ and that $A, B$ are finite sets. We let $T: U \rightarrow W$ be a linear transformation mapping nonzero decomposable tensors into nonzero decomposable tensors.

Let $S_{a}$ and $R_{b}$ be the sets of one dimensional subspaces of $U_{a}$ and $W_{b}$ respectively. We define a function $f: \Pi\left(S_{a}: a \in A\right) \rightarrow \Pi\left(R_{b}: b \in B\right)$ as follows. Let $x_{a} \in U_{a}$ be nonzero and let $T\left(\otimes\left(x_{a}: a \in A\right)\right)=\otimes\left(y_{b}: b \in B\right)$. Let $x \in \Pi\left(S_{a}: a \in A\right)$ and $y \in \Pi\left(R_{b}: b \in B\right)$ such that $p_{a}(x)=\left\langle x_{a}\right\rangle$ and $p_{b}(y)=\left\langle y_{b}\right\rangle$. We set $f(x)=y$ and note that by the above lemma, $f$ is well defined. We prove next the 
3.2. Lemma. The function $f$ above is an adjacency preserving function.

Proof. Let $u, u^{\prime} \in \Pi\left(S_{a}: a \in A\right)$ be adjacent and choose $x_{a}, x_{a}^{\prime} \in u_{a}$ such that $p_{a}(u)=\left\langle x_{a}\right\rangle, p_{a}\left(u^{\prime}\right)=\left\langle x_{a}^{\prime}\right\rangle$. Let $x=\otimes\left(x_{a}: a \in A\right)$ and $x^{\prime}=$ $\otimes\left(x_{a}^{\prime}: a \in A\right)$. Then, by $(2)(b)$ of Lemma $3.1, x+x^{\prime}$ is a decomposable tensor and by (2)(a) we have that $\langle x\rangle \neq\left\langle x^{\prime}\right\rangle$. Let $y=T(x)=$ $\otimes\left(y_{b}: b \in B\right)$ and $y^{\prime}=T\left(x^{\prime}\right)=\otimes\left(y_{b}^{\prime}: b \in B\right)$. Then $y+y^{\prime}$ is a decomposable tensor. Let $w, w^{\prime} \in \Pi\left(R_{b}: b \in B\right)$ be such that $p_{b}(w)=\left\langle y_{b}\right\rangle$ and $p_{b}\left(w^{\prime}\right)=\left\langle y_{b}^{\prime}\right\rangle$. Then $w$ and $w^{\prime}$ are either adjacent or equal. If they were equal then $y=e y^{\prime}$ for some $e \in F$, from which we get $T\left(x-e x^{\prime}\right)=0$, a contradiction since $x-e x^{\prime}$ is a nonzero decomposable tensor for all $e \in F$.

3.3. Definition. For each $A^{\prime} \varsubsetneqq A$ and $x \in \Pi\left(U_{a}: a \notin A^{\prime}\right)$ we define a multilinear function $N_{x}: \Pi\left(U_{a}: a \in A^{\prime}\right) \rightarrow U$ by setting $N_{x}(u)=$ $\otimes\left(v_{a}: a \in A\right)$ where $v_{a}=p_{a}(x)$ for $a \notin A^{\prime}$ and $v_{a}=p_{a}(u)$ for $a \in A^{\prime}$. We let $M_{x}: \otimes\left(U_{a}: a \in A^{\prime}\right) \rightarrow U$ be the linear transformation induced by $N_{x}$.

Since $\operatorname{dim}\left(U_{a}\right) \geqq 2$, each $S_{a}$ contains at least three elements, and therefore we can apply Lemma 2.4 to obtain the function $\sigma: A \rightarrow B$ satisfying the conclusions of that lemma. Let $\sigma(A)=\left\{b_{1}, \cdots, b_{k}\right\}$ and $A_{i}=\sigma^{-1}\left(b_{i}\right)$. Let $V_{i}=\otimes\left(U_{a}: a \in A_{i}\right), \quad V=\otimes\left(V_{i}: i=1, \cdots, k\right)$ and let $\varphi: U \rightarrow V$ be the canonical isomorphism.

3.4. THEOREM. The decomposable tensor preserver $T$ has the form $M_{y} \cdot\left(T_{1} \otimes \cdots \otimes T_{k}\right) \cdot \varphi$ where

(1) $y \in \Pi\left(W_{b}: b \notin \sigma(A)\right)$, or $M_{y}$ is deleted if $\sigma(A)=B$,

(2) $T_{i}: V_{i} \rightarrow W_{b_{i}}$ is a linear transformation mapping nonzero decomposable tensors of $V_{i}$ into nonzero vectors of $W_{b_{i}}$.

Proof. Let $x_{i} \in \Pi\left(U_{a}: a \notin A_{i}\right)$ be chosen for $i=1, \cdots, k$. Consider $T \cdot M_{x_{i}}: V_{i} \rightarrow W$. For each $v \in V_{i}, M_{x_{i}}(v)=\bigotimes\left(v_{a}: a \in A\right)$ where $\left\langle v_{a}\right\rangle$ does not depend on $v$ whenever $a \notin A_{i}$. Therefore, since $\left(p_{b} \cdot f\right)(s)$, for $s \in \Pi\left(S_{a}: a \in A\right)$, does not depend on the coordinates of $s$ in $A \backslash \sigma^{-1}(b)$, there are fixed $w_{b} \in W_{b}$ for each $b \in B, b \neq b_{i}$, such that the image of $V_{i}$ under $T \cdot M_{x_{i}}$ has the form $\left\{\otimes\left(w_{b}^{\prime}: b \in B\right): w_{b}^{\prime}=w_{b}\right.$ for $b \neq b_{i}$ and $w_{b_{i}}^{\prime} \in W_{b_{i}}^{\prime}$, where $W_{b_{i}}^{\prime}$ is a subspace of $W_{b_{i}}$. Then $T \cdot M_{x_{i}}$ induces a linear transformation $T_{i}: V_{i} \rightarrow W_{b_{i}}$ defined by $T_{i}(v)=w_{b_{i}}^{\prime}$ where $T \cdot M_{x_{i}}(v)=\otimes\left(w_{b}^{\prime}: b \in B\right), w_{b}^{\prime}=w_{b}$ for $b \neq b_{i}$. If $x_{i}^{\prime} \in \Pi\left(U_{a}: a \notin A_{i}\right)$ is another element and $T_{i}^{\prime}: V_{i} \rightarrow W_{b_{i}}$ is induced as above by $T \cdot M_{x_{i}^{\prime}}$, then for each decomposable tensor $x \in V_{i}$ there is a $c_{x} \in F$ such that $T_{i}(x)=$ $c_{x} T_{i}^{\prime}(x)$. It then follows easily that there is a $c \in F$ such that $T_{i}=c T_{i}^{\prime}$.

By Theorem 2.5 the image of $\Pi\left(S_{a}: a \in A\right)$ under $f$ is $\Pi\left(Q_{b}: b \in B\right)$ where $Q_{b} \subseteq R_{b}$ can be given explicitly. For each $b \notin \sigma(A), Q_{b}$ consists 
of one element and we let $Q_{b}=\left\langle y_{b}\right\rangle$. Let $y \in \Pi\left(W_{b}: b \notin \sigma(A)\right)$ where $p_{b}(y)=y_{b}$, and let $T^{\prime}=M_{y} \cdot\left(T_{1} \otimes \cdots \otimes T_{k}\right) \cdot \varphi$. If $x \in U$ is a decomposable tensor then $T(x)=e_{x} T^{\prime}(x)$ for some $e_{x} \in F$, and therefore it follows that $T=e T^{\prime}$ on all of $U$ for some $e \in F$.

3.5. THEOREM. A decomposable tensor preserve of $U$ onto itself has the form $\otimes\left(T_{a}: a \in A\right)$ where $T_{a}: U_{a} \rightarrow U_{\sigma(a)}$ is an onto nonsingular linear transformation and $\sigma: A \rightarrow A$ is a permutation.

Proof. Let $f: \Pi\left(S_{a}: a \in A\right) \rightarrow \Pi\left(S_{a}: a \in A\right)$ be the adjacency preserving function induced by the decomposable tensor preserver and let $\sigma: A \rightarrow A$ be the function induced by $f$. Then the image of $\Pi\left(S_{a}: a \in A\right)$ under $f$ has the form $\Pi\left(Q_{a}: a \in A\right)$ and therefore the image of $U$ is spanned by elements $\otimes\left(u_{a}: a \in A\right)$ where $u_{a}$ belongs to the smallest subspace of $U_{a}$ which contains all the subspaces making up $Q_{a}$. Since $\operatorname{dim}\left(U_{a}\right) \geqq 2$ and the tensor preserver is assumed to be onto, no $Q_{a}$ can consist of only one element. Therefore $\sigma$ is a permutation. The theorem now follows from Theorem 3.4. That $T_{a}$ is onto and nonsingular is clear.

3.6. Definition. If $V$ is a vector space over a field $F$ and if $\mathscr{L}(V)$ is the vector space of linear transformations of $V$ into itself, then a subspace of $\mathscr{L}(V)$ is called a nonsingular subspace if each of its nonzero elements is a nonsingular linear transformation.

3.7. ThEOREM. Let $k \geqq 2$ be an integer and let $W_{1}, \cdots, W_{k}$ be vector spaces over a field $F$ where $2 \leqq \operatorname{dim}\left(W_{1}\right) \leqq \cdots \leqq \operatorname{dim}\left(W_{k}\right)<\infty$. Then there is a linear transformation $L: \otimes\left(W_{i}: i=1, \cdots, k\right) \rightarrow W_{k}$ mapping nonzero decomposable tensors into nonzero vectors if and only if $\mathscr{L}\left(W_{k}\right)$ contains a nonsingular subspace with dimension equal to $\operatorname{dim}\left(W_{k-1}\right)$.

Proof. Suppose that $L$ exists. Let $w_{i} \in W_{i}$ for $i=1, \cdots, k-2$ be nonzero vectors. For each $x \in W_{k-1}$ we let

$$
\mathscr{W}_{x}=\left\{w_{1} \otimes \cdots \otimes w_{k-2} \otimes x \otimes y: y \in W_{k}\right\},
$$

and note that $\mathscr{W}_{x}$ is a subspace consisting of decomposable tensors. Let $L_{x}=\left(L \mid \mathscr{W}_{x}\right)$ and let $I_{x}: W_{k} \rightarrow \mathscr{W}_{x}$ be defined by

$$
I_{x}(y)=w_{1} \otimes \cdots \otimes w_{k-2} \otimes x \otimes y
$$

for $y \in W_{k}$. Then $L_{x} I_{x} \in \mathscr{L}\left(W_{k}\right)$ is nonsingular for $x \neq 0$. Let $\operatorname{dim}\left(W_{k-1}\right)=t$ and let $\left\{x_{1}, \cdots, x_{t}\right\}$ be a basis of $W_{k-1}$. Then $\left\{L_{x_{1}} I_{x_{1}}, \cdots, L_{x_{t}} I_{x_{t}}\right\}$ spans a nonsingular subspace of $\mathscr{L}\left(W_{k}\right)$. For, 
suppose $\sum_{i=1}^{t} a_{i} L_{x_{i}} I_{x_{i}}$ is singular. Choose $y \neq 0$ such that

$$
\sum_{i=1}^{t} a_{i} L_{x_{i}} I_{x_{i}}(y)=0
$$

Then, since $\sum_{i=1}^{t} a_{i} L_{x_{i}} I_{x_{i}}(y)=L\left(w_{1} \otimes \cdots \otimes w_{k-2} \otimes \sum_{i=1}^{t} a_{i} x_{i} \otimes y\right)$, we must have that $\sum_{i=1}^{t} a_{i} x_{i}=0$. Therefore $a_{1}=\cdots=a_{t}=0$.

Suppose that $\mathscr{L}\left(W_{k}\right)$ has a nonsingular subspace with dimension $t=\operatorname{dim}\left(W_{k-1}\right)$. We construct $L$ inductively. Suppose that

$$
L_{0}: W_{2} \otimes \cdots \otimes W_{k} \rightarrow W_{k}
$$

has been defined such that nonzero decomposable tensors of $W_{2} \otimes \cdots \otimes W_{k}$ are mapped into nonzero vectors of $W_{k}$. Let $s=$ $\operatorname{dim}\left(W_{1}\right)$ and let $\left\{L_{1}, \cdots, L_{s}\right\}$ be a basis of an $s$-dimensional nonsingular subspace of $\mathscr{L}\left(W_{k}\right)$. Such a basis exists since $s=\operatorname{dim}\left(W_{1}\right) \leqq$ $\operatorname{dim}\left(W_{k-1}\right)$. Let $\left\{x_{1}, \cdots, x_{s}\right\}$ be a basis of $W_{1}$. Let $N: W_{1} \otimes W_{k} \rightarrow W_{k}$ be the linear transformation induced by the multilinear function $\bar{N}: W_{1} \times W_{l} \rightarrow W_{k}$ where $\bar{N}\left(\sum_{i=1}^{s} a_{i} x_{i}, y\right)=\sum_{i=1}^{s} a_{i} L_{i}(y)$. Then $\bar{N}(x, y)=0$ implies that $x=0$ or $y=0$ and therefore $N(x \otimes y)=0$ if and only if $x \otimes y=0$. Let $I: W_{1} \rightarrow W_{1}$ be the identity and let $L=N \cdot\left(I \otimes L_{0}\right)$. Then $L\left(w_{1} \otimes \cdots \otimes w_{k}\right)=N\left(w_{1} \otimes L_{0}\left(w_{2} \otimes \cdots \otimes w_{k}\right)\right)=0$ implies that $w_{1}=0 \quad$ or $L_{0}\left(w_{2} \otimes \cdots \otimes w_{k}\right)=0$. Therefore, either $w_{1}=0$ or $w_{2} \otimes \cdots \otimes w_{k}=0$, and in both cases $w_{1} \otimes \cdots \otimes w_{k}=0$. This completes the proof.

3.8. Theorem. Let $F$ be algebraically closed and let $T: U \rightarrow U$ be a decomposable tensor preserver where $\operatorname{dim}\left(U_{a}\right)$ is finite for all $a \in A$. Then $T=\otimes\left(T_{a}: a \in A\right)$ where $T_{a}: U_{a} \rightarrow U_{\sigma(a)}$ is a nonsingular linear transformation and $\sigma: A \rightarrow A$ is a permutation satisfying $\operatorname{dim}\left(U_{a}\right)=\operatorname{dim}\left(U_{\sigma(a)}\right)$ for $a \in A$.

Proof. We prove that $\sigma$ is a permutation. By Theorem 3.4, $T=M_{y} \cdot\left(T_{1} \otimes \cdots \otimes T_{k}\right) \cdot \varphi$ where the domain of $T_{i}$ is $V_{i}=\otimes\left(U_{a}: a \in A_{i}\right)$ and $A_{i}=\sigma^{-1}\left(a_{i}\right)$ for some $a_{i} \in A$. For each $a \in A_{i}, V_{\imath}$ contains a subspace with dimension equal to $\operatorname{dim}\left(U_{a}\right)$ which consists of decomposable tensors only. It follows that $U_{a_{i}}$, which is the range space of $V_{i}$ under $T_{i}$, has dimension at least as large as the maximum of the $\operatorname{dim}\left(U_{a}\right)$ for $a \in A_{i}$. Therefore, for each $a \in A, \operatorname{dim}\left(U_{a}\right) \leqq \operatorname{dim}\left(U_{\sigma(a)}\right)$. Suppose that $\sigma$ is not one-to-one. Of those $a \in A$ for which $\sigma^{-1}(\sigma(a))$ consists of at least two elements, choose one, say $b$, for which $\operatorname{dim}\left(U_{b}\right)$ is maximal. Then $\operatorname{dim}\left(U_{b}\right)=\operatorname{dim}\left(U_{\sigma(b)}\right)$. For, suppose that $\operatorname{dim}\left(U_{b}\right)<\operatorname{dim}\left(U_{\sigma(b)}\right)$. Then $\sigma$ maps the set $\left\{a \mid \operatorname{dim}\left(U_{a}\right)>\operatorname{dim}\left(U_{b}\right)\right\} \cup\{b\}$ into the set $\left\{a \mid \operatorname{dim}\left(U_{a}\right)>\operatorname{dim}\left(U_{b}\right)\right\}$, and consequently, there is a $b^{\prime} \in A$ for which $\sigma^{-1}\left(\sigma\left(b^{\prime}\right)\right)$ has at least two elements and $\operatorname{dim}\left(U_{b^{\prime}}\right)>\operatorname{dim}\left(U_{b}\right)$. 
This contradicts the choice of $b$. Now, $\sigma(b) \in \sigma(A)$ so that $\sigma(b)=a_{i}$ for some $i$ and $T_{i}: V_{i} \rightarrow U_{a_{i}}$. By Theorem 3.7, $\mathscr{L}\left(U_{a_{i}}\right)$ contains a nonsingular subspace with dimension at least two. This is impossible since $F$ is assumed to be algebraically closed (for nonsingular $C$ and $D, C-e D$ is singular for any eigenvalue $e$ of $D^{-1} C$ ). Therefore $\sigma$ is a permutation and the theorem follows from Theorem 3.4.

\section{REFERENCE}

1. Marvin Marcus and B. N. Moyls, Transformations on tensor product spaces, Pacific

J. Math. 9 (1959), 1215-1221.

Received May 2, 1966.

UNIVERsity OF BRITISH COLumbia 


\section{PACIFIC JOURNAL OF MATHEMATICS}

\section{H. ROYDEN}

Stanford University

Stanford, California

\author{
J. P. JANS \\ University of Washington \\ Seattle, Washington 98105
}

\section{EDITORS}

\author{
J. DugundJI \\ Department of Mathematics \\ Rice University \\ Houston, Texas 77001 \\ Richard ARENS \\ University of California \\ Los Angeles, California 90024
}

\section{ASSOCIATE EDITORS}
E. F. BECKENBACH
B. H. NeumanN
F. WOLF
K. YOSIDA

\section{SUPPORTING INSTITUTIONS}

\author{
UNIVERSITY OF BRITISH COLUMBIA \\ CALIFORNIA INSTITUTE OF TECHNOLOGY \\ UNIVERSITY OF CALIFORNIA \\ MONTANA STATE UNIVERSITY \\ UNIVERSITY OF NEVADA \\ NEW MEXICO STATE UNIVERSITY \\ OREGON STATE UNIVERSITY \\ UNIVERSITY OF OREGON \\ OSAKA UNIVERSITY \\ UNIVERSITY OF SOUTHERN CALIFORNIA
}

\author{
STANFORD UNIVERSITY \\ UNIVERSITY OF TOKYO \\ UNIVERSITY OF UTAH \\ WASHINGTON STATE UNIVERSITY \\ UNIVERSITY OF WASHINGTON \\ AMERICAN MATHEMATICAL SOCIETY \\ CHEVRON RESEARCH CORPORATION \\ TRW SYSTEMS \\ NAVAL ORDNANCE TEST STATION
}

Mathematical papers intended for publication in the Pacific Journal of Mathematics should be typewritten (double spaced). The first paragraph or two must be capable of being used separately as a synopsis of the entire paper. It should not contain references to the bibliography. Manuscripts may be sent to any one of the four editors. All other communications to the editors should be addressed to the managing editor, Richard Arens at the University of California, Los Angeles, California 90024.

50 reprints per author of each article are furnished free of charge; additional copies may be obtained at cost in multiples of 50 .

The Pacific Journal of Mathematics is published monthly. Effective with Volume 16 the price per volume (3 numbers) is $\$ 8.00$; single issues, $\$ 3.00$. Special price for current issues to individual faculty members of supporting institutions and to individual members of the American Mathematical Society: $\$ 4.00$ per volume; single issues $\$ 1.50$. Back numbers are available.

Subscriptions, orders for back numbers, and changes of address should be sent to Pacific Journal of Mathematics, 103 Highland Boulevard, Berkeley 8, California.

Printed at Kokusai Bunken Insatsusha (International Academic Printing Co., Ltd.), 7-17, Fujimi 2-chome, Chiyoda-ku, Tokyo, Japan.

PUBLISHED BY PACIFIC JOURNAL OF MATHEMATICS, A NON-PROFIT CORPORATION

The Supporting Institutions listed above contribute to the cost of publication of this Journal, but they are not owners or publishers and have no responsibility for its content or policies. 


\section{Pacific Journal of Mathematics}

A. A. Aucoin, Diophantine systems ............................. 419

Charles Ballantine, Products of positive definite matrices. I ............... 427

David Wilmot Barnette, A necessary condition for d-polyhedrality ............ 435

James Clark Beidleman and Tae Kun Seo, Generalized Frattini subgroups of finite groups ......................................... 441

Carlos Jorge Do Rego Borges, A study of multivalued functions ............. 451

William Edwin Clark, Algebras of global dimension one with a finite ideal

lattice ...............................................

Richard Brian Darst, On a theorem of Nikodym with applications to weak convergence and von Neumann algebras .........................

George Wesley Day, Superatomic Boolean algebras .....................

Lawrence Fearnley, Characterization of the continuous images of all

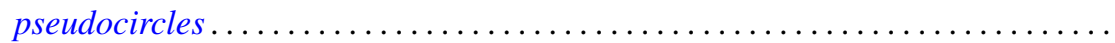

Neil Robert Gray, Unstable points in the hyperspace of connected subsets....... 515

Franklin Haimo, Polynomials in central endomorphisms .................. 521

John Sollion Hsia, Integral equivalence of vectors over local modular lattices . . . . 527

Jim Humphreys, Existence of Levi factors in certain algebraic groups .......... 543

E. Christopher Lance, Automorphisms of postliminal $C^{*}$-algebras ............ 547

Sibe Mardesic, Images of ordered compacta are locally peripherally metric . . . . 557

Albert W. Marshall, David William Walkup and Roger Jean-Baptiste Robert Wets,

Order-preserving functions: Applications to majorization and order

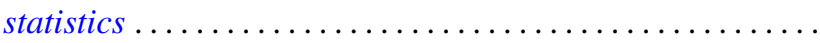

Wellington Ham Ow, An extremal length criterion for the parabolicity of

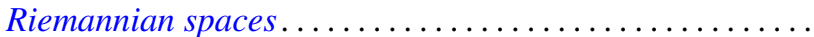

585

Wellington Ham Ow, Criteria for zero capacity of ideal boundary components of

Riemannian spaces...................................... 591

J. H. Reed, Inverse limits of indecomposable continua .................. 597

Joseph Gail Stampfli, Minimal range theorems for operators with thin spectra . . . 601

Roy Westwick, Transformations on tensor spaces..................... 613

Howard Henry Wicke, The regular open continuous images of complete metric

spaces ........................................... 621

Abraham Zaks, A note on semi-primary hereditary rings .............. 627

Thomas William Hungerford, Correction to: "A description of $\operatorname{Mult}_{i}\left(A^{1}, \cdots, A^{n}\right)$

by generators and relations" ............................. 629

Uppuluri V. Ramamohana Rao, Correction to: "On a stronger version of Wallis' formula".............................................. 629

Takesi Isiwata, Correction: "Mappings and spaces" ................... 630

Henry B. Mann, Josephine Mitchell and Lowell Schoenfeld, Correction to:

"Properties of differential forms in $n$ real variables" .... . .

James Calvert, Correction to: "An integral inequality with applications to the

Dirichlet problem"............................. 